\title{
Formation of Singularities in Three-Dimensional Compressible Fluids
}

\author{
Thomas C. Sideris \\ Department of Mathematics, University of California, Santa Barbara, California 93106, USA
}

\begin{abstract}
Presented are several results on the formation of singularities in solutions to the three-dimensional Euler equations for a polytropic, ideal fluid under various assumptions on the initial data. In particular, it is shown that a localized fluid which is initially compressed and outgoing, on average, will develop singularities regardless of the size of the initial disturbance.
\end{abstract}

This article presents a number of results on formation of singularities in solutions to the three-dimensional compressible Euler equations for a polytropic, ideal fluid. The results, described precisely in the following section, show that $C^{1}$ solutions to the equations do not exist for all time, under various restrictions on the initial data.

Theorems one and two, below, deal with the case of "large" data which essentially means that the initial flow velocity must be supersonic in some region relative to the sound speed at infinity. (The initial data is constant outside a bounded set.) Singularity formation is detected as the disturbance overtakes the wave front (presumably as a shock wave) forcing the front to propagate with supersonic speed. The method, which is a refinement of [10], applies equally well in one and two space dimensions.

Our main result, Theorem 3, shows that a fluid will develop singularities if, on average, it is slightly compressed and out-going near the wave front. The proof borrows some important technical points from an earlier result on nonlinear wave equations in three space dimensions [9].

Although the extensive one-dimensional theory $[2,5,7,8]$ strongly suggests such results, the customary method of characteristics has not proved tractable in higher dimensions. (John [3] has recently used characteristics to prove blow-up for a threedimensional scalar wave equation with spherical symmetry.) Our approach involves the use of averaged quantities, thereby avoiding for the most part, local analysis of the solution. For classical solutions, these averages satisfy certain differential inequalities, solutions of which have finite life span.

We do not address the problem of singularity formation in three-dimensional incompressible fluids. It is possible to approximate a compressible flow with an 
incompressible one by letting the sound speed become large [6]. However, our upper bound for the life span of a $C^{1}$ compressible flow is not uniform in this parameter, and so this approximation cannot be used to establish singularity formation in the incompressible case.

\section{Statement of Results}

The motion of a polytropic, ideal gas is described by the compressible Euler equations [1]:

$$
\begin{array}{r}
\rho_{t}+\nabla \cdot \rho u=0, \\
\rho\left(u_{t}+u \cdot \nabla u\right)+\nabla p=0, \\
S_{t}+u \cdot \nabla S=0, \\
p=A \rho^{\gamma} e^{S} \quad(A>0, \gamma>1) .
\end{array}
$$

Here, $\rho, u, S, p$ represent the density, velocity, specific entropy, and pressure of the gas, respectively. The state Eq. (1.1d) is that of a polytropic gas, $\gamma$ being the adiabatic index.

Initial data is assigned which is constant outside the bounded set $\{|x| \geqq R\}$ :

$$
\begin{aligned}
& \rho(x, 0)=\rho^{0}(x)>0 ; \quad \rho^{0}(x)=\bar{\rho}, \quad|x| \geqq R, \\
& u(x, 0)=u^{0}(x) ; \quad u^{0}(x)=\bar{u}(=0), \quad|x| \geqq R, \\
& S(x, 0)=S^{0}(x) ; \quad S^{0}(x)=\bar{S}, \quad|x| \geqq R .
\end{aligned}
$$

By making the change of variables $u \rightarrow u-\bar{u}$ and $x \rightarrow x+t \bar{u}$, we may assume without further loss of generality that $\bar{u}=0$, as indicated in (1.2b).

The Eqs. $(1.1 \mathrm{a}-\mathrm{d})$ can be written as a positive, symmetric hyperbolic system, and as such, they possess a unique, local $C^{1}$ solution $(\rho, u, S)$ with $\rho>0$, provided the initial data $(1.2 \mathrm{a}-\mathrm{c})$ are sufficiently regular [4].

The maximum speed of propagation of the front of a smooth disturbance is governed by the sound speed

$$
\sigma=p_{\rho}(\bar{\rho}, \bar{S})^{1 / 2}=\left[A \gamma \bar{\rho}^{\gamma-1} e^{\bar{S}}\right]^{1 / 2},
$$

since $\bar{u}=0$. More precisely, letting

$$
D(t)=\left\{x \in \mathbb{R}^{3}:|x| \geqq R+\sigma t\right\},
$$

we have the following.

Proposition. If $(\rho, u, S)$ is a $C^{1}$ solution of $(1.1 a-d),(1.2 a-c)$ on $D(t)$, for $0 \leqq t \leqq T$, then $(\rho, u, S) \equiv(\bar{\rho}, 0, \bar{S})$ on $D(t), 0 \leqq t<T$.

This is a consequence of local energy estimates; see the proposition in [10].

Our first result on formation of singularities, arising from "large" initial disturbances, relies on the relationship among the quantities

$$
m(t)=\int_{\mathbb{R}^{3}}(\rho(x, t)-\bar{\rho}) d x,
$$




$$
\begin{aligned}
& \eta(t)=\int_{\mathbb{R}^{3}}[\rho(x, t) \exp (S(x, t) / \gamma)-\bar{\rho} \exp (\bar{S} / \gamma)] d x, \\
& F(t)=\int_{\mathbb{R}^{3}} x \cdot \rho u(x, t) d x,
\end{aligned}
$$

which, roughly speaking, measure mass, entropy, and the radial component of momentum. These integrals converge as long as the solution is $C^{1}$, by the proposition.

Theorem 1. Suppose $(\rho, u, S)$ is a $C^{1}$ solution of $(1.1 a-d),(1.2 a-c)$ for $0 \leqq t \leqq T$. If

$$
\begin{aligned}
& m(0) \geqq 0 \\
& \eta(0) \geqq 0 \\
& F(0) \geqq \alpha \sigma R^{4} \max \rho^{0}(x) \quad\left(\alpha=\frac{16 \pi}{3}\right)
\end{aligned}
$$

then the life span $T$ of the $C^{1}$ solution is finite.

To illustrate one way in which $(1.5 \mathrm{a}-\mathrm{c})$ can be satisfied, take as initial conditions

$$
\rho^{0} \equiv \bar{\rho} \quad \text { and } \quad S^{0} \equiv \bar{S} .
$$

Then $m(0)=\eta(0)=0$ and $(1.5 \mathrm{c})$ holds if

$$
\int_{\mathbb{R}^{3}} x \cdot u^{0}(x) d x \geqq \alpha \sigma R^{4} .
$$

Comparing both sides, we find that the initial flow velocity must be supersonic in some region.

The system (1.1 a-d) may also be described in terms of the energy $E$, rather than the specific entropy $S$. Thus, (1.1c) is replaced by the energy conservation law

$$
E_{t}+\nabla \cdot\left[u\left(\gamma E-\frac{\gamma-1}{2} \rho|u|^{2}\right)\right]=0,
$$

the new state equation is

$$
p=(\gamma-1)\left[E-\frac{1}{2} \rho|u|^{2}\right],
$$

and $E$ must be prescribed initially instead of $S$

$$
E(x, 0)=E^{0}(x)>0 ; \quad E^{0}(x)=\bar{E}, \quad|x| \geqq R .
$$

A $C^{1}$ solution of $(1.1 \mathrm{a}-\mathrm{d}),(1.2 \mathrm{a}-\mathrm{c})$ gives rise to a $C^{1}$ solution of $\left(1.1 \mathrm{a}, \mathrm{b}, \mathrm{c}^{\prime}, \mathrm{d}^{\prime}\right)$, $\left(1.2 \mathrm{a}, \mathrm{b}, \mathrm{c}^{\prime}\right)$ through the relation

$$
E=\frac{1}{2} \rho|u|^{2}+\frac{A}{\gamma-1} \rho^{\gamma} e^{S} .
$$

Thus, Theorem 1 has an interpretation in this situation, as well. Moreover, defining

$$
e(t)=\int_{\mathbb{R}^{3}}(E(x, t)-\bar{E}) d x,
$$


we will also prove a slightly different "large data" result:

Theorem 2. Suppose $(\rho, u, E)$ is a $C^{1}$ solution of $\left(1.1 a, b, c^{\prime}, d^{\prime}\right),\left(1.2, a, b, c^{\prime}\right)$ for $0 \leqq t \leqq T$. If

$$
\begin{aligned}
m(0) & \geqq 0, \\
e(0) & \geqq 0, \\
1 & <\gamma<5 / 3^{\star}, \\
F(0) & \geqq \beta \sigma R^{4} \max \rho^{0}(x) \quad\left(\beta=\frac{32 \pi}{3(\gamma-1)}\right),
\end{aligned}
$$

then the life span $T$ is finite.

Our main result establishes formation of singularities without any condition of largeness such as $(1.5 \mathrm{c})$ or $(1.6 \mathrm{~d})$. Let us define the functions

$$
\begin{aligned}
& q^{0}(r)=\int_{|x|>r}|x|^{-1}(|x|-r)^{2}\left(\rho^{0}(x)-\bar{\rho}\right) d x, \\
& q^{1}(r)=\int_{|x|>r}|x|^{-3}\left(|x|^{2}-r^{2}\right) x \cdot \rho^{0} u^{0}(x) d x .
\end{aligned}
$$

Theorem 3. Suppose that for some $0<R_{0}<R$

$$
\begin{aligned}
& q^{0}(r)>0, \\
& q^{1}(r) \geqq 0,
\end{aligned}
$$

for $R_{0}<r<R$, and suppose

$$
S^{0}(x) \geqq \bar{S} .
$$

Then the life span $T$ of the $C^{1}$ solution of $(1.1 a-d),(1.2 a-c)$ is finite.

The assumptions $(1.7 \mathrm{a}, \mathrm{b})$ mean that, in an average sense, the gas must be slightly compressed and outgoing directly behind the wave front. It is interesting to observe that both $q^{0}$ and $q^{1}$ vanish for incompressible data $\rho^{0}=\bar{\rho}, \nabla \cdot u^{0}=0$ (by the divergence theorem in the case of $q^{1}$ ). Thus, singularities due to compression are being detected, and not those of the underlying incompressible flow, if indeed any are present.

To indicate the idea of the proof, suppose $S^{0}(x) \equiv \bar{S}$ and $\gamma=2$. Disregarding the fact that the solution is only assumed to be $C^{1}$, we derive the second order acoustical wave equation

$$
(\rho-\bar{\rho})_{t t}-\sigma^{2} \Delta(\rho-\bar{\rho})=A e^{\bar{s}} \Delta(\rho-\bar{\rho})^{2}+\sum_{i, j=1}^{3} \frac{\partial^{2}}{\partial x_{i} \partial x_{j}}\left(\rho u_{i} u_{j}\right),
$$

from $(1.1 \mathrm{a}-\mathrm{d})$. By performing appropriate averages, the last term may be ignored, leading to an inequality of the form

$$
v_{t t}-\Delta v \geqq \Delta v^{2}
$$

* The elementary kinetic theory of gases holds in the range $1<\gamma \leqq 5 / 3$ 
solutions of which develop singularities for small data [9]. The proof relies on the specific form of the Riemann function for the wave equation in three dimensions, in particular, its positivity. This contrasts with the results of theorems one and two which are dimension independent.

\section{Proof of Theorems 1 and 2}

Let $(\rho, u, S)$ be a $C^{1}$ solution of $(1.1 \mathrm{a}-\mathrm{d}),(1.2 \mathrm{a}-\mathrm{c})$ for $0 \leqq t<T$. Using the proposition and integration by parts it is easily shown that

and

$$
m(t)=m(0)
$$

$$
\eta(t)=\eta(0)
$$

Similarly, we derive

$$
F^{\prime}(t)=\int\left[\rho|u|^{2}+3(p-\bar{p})\right] d x
$$

where $\bar{p}=p(\bar{\rho}, \bar{S})$.

The goal is to obtain a differential inequality for $F(t)$.

Define $B(t)=\{x:|x| \leqq R+\sigma t\}=D(t)^{c}$. The proposition says that $(\rho, u, S)=$ $(\bar{\rho}, 0, \bar{S})$ outside $B(t)$. By (1.1d), Jensen's inequality, $(2.1 \mathrm{~b})$, and (1.5b) we have

$$
\begin{aligned}
\int_{B(t)} p d x & =A \int_{B(t)} \rho^{\gamma} \exp S d x \\
& \geqq A(\operatorname{vol} B(t))^{1-\gamma}\left(\int_{B(t)} \rho \exp (S / \gamma) d x\right)^{\gamma} \\
& =A(\operatorname{vol} B(t))^{1-\gamma}(\eta(0)+\operatorname{vol} B(t) \bar{\rho} \exp (\bar{S} / \gamma))^{\gamma} \\
& \geqq \bar{p} \operatorname{vol} B(t) \\
& =\int_{B(t)} \bar{p} d x
\end{aligned}
$$

Returning to (2.1c), this gives

$$
F^{\prime}(t) \geqq \int \rho|u|^{2} d x
$$

Next, we apply Schwartz' inequality to obtain

$$
F(t)^{2}=\left(\int_{B(t)} x \cdot \rho u d x\right)^{2} \leqq\left(\int_{B(t)}|x|^{2} \rho d x\right)\left(\int_{B(t)} \rho|u|^{2} d x\right) .
$$

With the aid of (2.1a) we have

$$
\begin{aligned}
\int_{B(t)}|x|^{2} \rho d x & \leqq(R+\sigma t)^{2} \int_{B(t)} \rho d x \\
& =(R+\sigma t)^{2}\left(m(0)+\int_{B(t)} \bar{\rho} d x\right) \\
& =(R+\sigma t)^{2}\left(\int_{B(t)}\left(\rho^{0}-\bar{\rho}\right) d x+\int_{B(t)} \bar{\rho} d x\right) \\
& \leqq \frac{4 \pi}{3}(R+\sigma t)^{5} \max \rho^{0}(x) .
\end{aligned}
$$


(2.2 a, b, c) combine to give the inequality

$$
F^{\prime}(t) \geqq\left[\frac{4 \pi}{3}(R+\sigma t)^{5} \max \rho^{0}(x)\right]^{-1} F(t)^{2},
$$

$F(0)>0$, so (2.3) implies that $F(t)$ remains positive on $0 \leqq t<T$. Dividing by $F(t)^{2}$ and integrating from 0 to $T$ in (2.3) results in

$$
F(0)^{-1} \geqq F(0)^{-1}-F(T)^{-1} \geqq\left(\alpha \sigma \max \rho^{0}\right)^{-1}\left(R^{-4}-(R+\rho T)^{-4}\right)
$$

with $\alpha=16 \pi / 3$. It is now clear that $T$ cannot become arbitrarily large without contradicting the assumption $(1.5 \mathrm{c})$. This proves Theorem 1.

In order to prove Theorem 2, suppose that $(\rho, u, E)$ is a $C^{1}$ solution of $\left(1.1 \mathrm{a}, \mathrm{b}, \mathrm{c}^{\prime}, \mathrm{d}^{\prime}\right),\left(1.2 \mathrm{a}, \mathrm{b}, \mathrm{c}^{\prime}\right)$ on $0 \leqq t<T$. Then $(2.1 \mathrm{c})$ is valid with $\bar{p}=p(\bar{\rho}, \bar{E})$. Thus, using $\left(1.1 \mathrm{~d}^{\prime}\right)$ and the fact that $e(t)=e(0)$ we obtain

$$
F^{\prime}(t)=\frac{5-3 \gamma}{2} \int\left[\rho|u|^{2}+3(\gamma-1) e(0)\right] d x .
$$

By $(1.6 \mathrm{~b}) e(0) \geqq 0$, so the remainder of the proof follows Theorem 1 , from (2.2a).

\section{Proof of Theorem 3}

Constants which are independent of the initial data will be denoted by $C$. They may depend on the fixed values $R_{0}$ and $R$, however. For the sake of clarity, we begin with the case $\gamma=2$, and later we will indicate what modifications are necessary in the general case.

Supposing $(\rho, u, S)$ is a $C^{1}$ solution, we have by the proposition that $\rho-\bar{\rho}$ is supported in $B(t)=\{|x| \leqq R+\sigma t\}$. So, we can define

where

$$
P(r, t)=\int_{|x|>r} \omega(x, r)(\rho(x, t)-\bar{\rho}) d x \quad(r>0),
$$

$$
\omega(x, r)=|x|^{-1}(|x|-r)^{2} .
$$

By direct computation, we have using (1.1a) that

$$
\begin{aligned}
\frac{\partial}{\partial t} P(r, t) & =\int_{|x|>r} \omega(x, r) \frac{\partial}{\partial t}(\rho(x, t)-\bar{\rho}) d x \\
& =-\int_{|x|>r} \omega(x, r) \nabla \cdot \rho u(x, t) d x \\
& =\int_{|x|>r} \nabla \omega(x, r) \cdot \rho u(x, t) d x,
\end{aligned}
$$

since $\rho u$ is supported in $B(t)$ and $\omega(x, r)=0$ on $\{|x|=r\}$. Thus, $P(r, t)$ is $C^{2}$ in $t$, and we can differentiate again using $(1.1 \mathrm{a}, \mathrm{b})$

$$
\begin{aligned}
\frac{\partial^{2}}{\partial t^{2}} P(r, t) & =\int_{|x|>r} \nabla \omega(x, r) \cdot \frac{\partial}{\partial t}(\rho u)(x, t) d x \\
& =-\sum_{i, j} \int_{|x|>r} \frac{\partial m}{\partial x_{i}}\left[\frac{\partial}{\partial x_{j}}\left(\rho u_{i} u_{j}\right)\right] d x-\int_{|x|>r} \nabla \omega \cdot \nabla(p-\bar{p}) d x
\end{aligned}
$$


where $\bar{p}=p(\bar{\rho}, \bar{S})$. Now since

$$
\nabla \omega(x, r)=|x|^{-3}\left(|x|^{2}-r^{2}\right) x
$$

which vanishes on $\{|x|=r\}$, and since $\rho u_{i} u_{j}$ and $p-\bar{p}$ have compact support, we may again integrate by parts:

$$
\begin{aligned}
\frac{\partial^{2}}{\partial t^{2}} P(r, t) & =\sum_{i, j} \int_{|x|>r} \frac{\partial^{2} \omega}{\partial x_{i} \partial x_{j}} \rho u_{i} u_{j} d x+\int_{|x|>r} \Delta \omega \cdot(p-\bar{p}) d x \\
& \equiv I_{1}(r, t)+I_{2}(r, t) .
\end{aligned}
$$

A simple computation of $\partial^{2} \omega / \partial x_{i} \partial x_{j}$ shows that

$$
\begin{aligned}
I_{1}(r, t) & =\int_{|x|>r} \frac{2 r^{2}}{|x|^{3}} \cdot \rho \cdot\left(\frac{x}{|x|} \cdot u\right)^{2} d x-\int_{|x|>r} \frac{|x|^{2}-r^{2}}{|x|^{3}} \cdot \rho \cdot\left(\frac{x}{|x|} \cdot u\right)^{2} d x \\
& +\int_{|x|>r} \frac{|x|^{2}-r^{2}}{|x|^{3}} \cdot \rho \cdot|u|^{2} d x \\
\geqq 0 &
\end{aligned}
$$

since $((x /|x|) \cdot u)^{2} \leqq|u|^{2}$.

For the second term, we have

$$
\Delta \omega(r, x)=2|x|^{-1}=\omega_{r r}(x, r) .
$$

Hence,

$$
I_{2}(r, t)=\int_{|x|>r} 2|x|^{-1}(p-\bar{p}) d x=\frac{\partial^{2}}{\partial r^{2}} \int_{|x|>r} \omega(x, r)(p(x, t)-\bar{p}) d x
$$

because $\omega$ and $\omega_{r}$ vanish on $\{|x|=r\}$. Combination of $(3.2 \mathrm{a}, \mathrm{b}, \mathrm{c})$ gives

$$
\left(\frac{\partial^{2}}{\partial t^{2}}-\sigma^{2} \frac{\partial^{2}}{\partial r^{2}}\right) P(r, t) \geqq G(r, t)
$$

where

$$
G(r, t) \equiv \frac{\partial^{2}}{\partial r^{2}} \int_{|x|>r} \omega(x, r)\left[p-\bar{p}-\sigma^{2}(\rho-\bar{\rho})\right] d x \equiv \frac{\partial^{2}}{\partial r^{2}} \widetilde{G}(r, t) .
$$

We may also write

$$
G(r, t)=\int_{|x|>r} 2|x|^{-1}\left[p-\bar{p}-\sigma^{2}(\rho-\bar{\rho})\right] d x .
$$

Inversion of the one dimensional d'Alembertian $\square=\partial^{2} / \partial t^{2}-\sigma^{2}\left(\partial^{2} / \partial r^{2}\right)$ gives (for $r>R_{0}+\sigma t$ )

$$
\begin{aligned}
P(r, t) & =P^{0}(r, t)+\frac{1}{2 \sigma} \int_{0}^{t} \int_{r-\sigma(t-\tau)}^{r+\sigma(t-\tau)} \square P(y, \tau) d y d \tau \\
& \geqq P^{0}(r, t)+\frac{1}{2 \sigma} \int_{0}^{t} \int_{r-\sigma(t-\tau)}^{r+\sigma(t-\tau)} G(y, \tau) d y d \tau,
\end{aligned}
$$


by $(3.3 \mathrm{a})$, where

$$
P^{0}(r, t)=\frac{1}{2}\left\{q^{0}(r+\sigma t)+q^{0}(r-\sigma t)+\frac{1}{\sigma} \int_{r}^{r+\sigma t} q^{1}(y) d y\right\} .
$$

Now let

$$
F(\mathrm{t})=\int_{0}^{t}(t-\tau) \int_{\sigma \tau+R_{0}}^{\sigma \tau+R} r^{-1} P(r, \tau) d r d \tau
$$

$F(t)$ is $C^{2}$, and from $(3.4 \mathrm{a})$,

$$
\begin{aligned}
F^{\prime \prime}(t) & =\int_{\sigma t+R_{0}}^{\sigma t+R} r^{-1} P(r, t) d r \\
& \geqq \int_{\sigma t+R_{0}}^{\sigma t+R} r^{-1} P^{0}(r, t) d r+\frac{1}{2 \sigma} \int_{\sigma t+R_{0}}^{\sigma t+R} r^{-1} \int_{0}^{t} \int_{r-\sigma(t-\tau)}^{r+\sigma(t-\tau)} G(y, \tau) d y d \tau d r \\
& \equiv J_{1}+J_{2} .
\end{aligned}
$$

By our hypotheses $(1.7 \mathrm{a}, \mathrm{b}), q_{0}(r)>0$ and $q_{1}(r) \geqq 0$ on $R_{0}<r<R$. Hence, we see from $(3.4 b)$ that

$$
J_{1} \geqq \int_{\sigma t+R_{0}}^{\sigma t+R} r^{-1} q^{0}(r-\sigma t) d r \geqq(\sigma t+R)^{-1} \int_{\sigma t+R_{0}}^{\sigma t+R} q^{0}(r-\sigma t) d r=B_{0}(\sigma t+R)^{-1}>0,
$$

where

$$
B_{0}=\int_{R_{0}}^{R} q^{0}(r) d r
$$

To bound $J_{2}$ below, we note that, as long as $u$ is $C^{1}$, the particle paths

$$
\frac{d x}{d t}=u(x, t) ; \quad x(0, \xi)=\xi
$$

exist. Equation (1.1c) expresses the fact that $S$ remains constant along these paths, so by $(1.7 \mathrm{c})$ we have that

$$
S(x, t) \geqq \bar{S}
$$

Consequently, $p(\rho, S) \geqq p(\rho, \bar{S})$, so that

$$
p-\bar{p}-\sigma^{2}(\rho-\bar{\rho}) \geqq A e^{\bar{S}}\left[\rho^{2}-\bar{\rho}^{2}-2 \bar{\rho}(\rho-\bar{\rho})\right]=A e^{\bar{S}}(\rho-\bar{\rho})^{2} .
$$

It follows from $(3.3 \mathrm{c})$ that

$$
G(r, t) \geqq 0 .
$$

Inverting the order of integration in $J_{2}$ (cf. (39a) in [9]) using the fact that $G(y, t)$ is supported in $\{y \leqq \sigma t+R\}$, we find

$$
J_{2}=\frac{1}{2 \sigma} \int_{0 \sigma \tau}^{t} \int_{R_{0}}^{\sigma \tau+R} G(y, \tau) \int_{\max \left[\sigma t+R_{0}, y-\sigma(t-\tau)\right]}^{y+\sigma(t-\tau)} r^{-1} d r d y d \tau,
$$

provided $t \geqq R_{1}=\frac{1}{2}\left(R-R_{0}\right)$. The innermost integral is bounded below by

$$
C \sigma(\sigma t+R)^{-2}(t-\tau)\left(y-\sigma \tau>R_{0}\right)^{2} .
$$


Since $G(y, \tau)>0$, we have that

$$
J_{2} \geqq C(\sigma t+R)^{-2} \int_{0 \sigma \tau+R_{0}}^{t} \int_{\tau \tau+R}^{R}(t-\tau)\left(y-\sigma \tau-R_{0}\right)^{2} G(y, \tau) d y d \tau,
$$

for $t \geqq R_{1}$. Returning to (3.3b), and noting that $\widetilde{G}(y, \tau)$ vanishes for $y>\sigma \tau+R$, we can integrate by parts and then use (3.7) to obtain

$$
\begin{aligned}
J_{2} & \geqq C(\sigma t+R)^{-2} \int_{0}^{t} \int_{\sigma \tau+R_{0}}^{\sigma \tau+R}(t-\tau) \widetilde{G}(y, \tau) d y d \tau \\
& \geqq C(\sigma t+R)^{-2} \frac{\sigma^{2}}{\bar{\rho}} \int_{0}^{t}(t-\tau) \int_{\sigma \tau+R_{0}}^{\sigma \tau+R} \int_{|x|>y} \omega(\rho-\bar{\rho})^{2} d x d y d \tau .
\end{aligned}
$$

Denoting this last integral by $J_{3}$ and using Schwartz's inequality, we find

$$
F(t)^{2} \leqq J_{3}\left(\int_{0}^{t}(t-t) \int_{\sigma \tau+R_{0}}^{\sigma \tau+R} y^{-2} \int_{y<|x|<\sigma \tau+R} \omega(x, y) d x d y d \tau\right)
$$

Letting $J_{4}$ denote the integral in (3.7b) above, we estimate as follows

$$
\begin{aligned}
J_{4} & =\int_{0}^{t}(t-\tau) \int_{\sigma \tau+R_{0}}^{\sigma \tau+R} y^{-2} 4 \pi \int_{y}^{\sigma \tau+R}|x|(|x|-y)^{2} d|x| d y d \tau \\
& \leqq C \int_{0}^{t}(t-\tau) \int_{\sigma \tau+R_{0}}^{\sigma \tau+R} y^{-2}(\sigma \tau+R)(\sigma \tau+R-y)^{3} d y d \tau \\
& \leqq C \int_{0}^{t}(t-\tau)(\sigma \tau+R) \int_{\sigma \tau+R_{0}}^{\sigma \tau+R} y^{-2} d y d \tau \\
& \leqq C \int_{0}^{t}(t-\tau)(\sigma \tau+R)^{-1} d \tau \\
& \leqq C \sigma^{-2}(\sigma t+R) \log (\sigma t+R) .
\end{aligned}
$$

Combining (3.8 a, b, c) and (3.5) we obtain

$$
F^{\prime \prime}(t) \geqq C \frac{\sigma^{4}}{\bar{p}}\left[(\sigma t+R)^{3} \log (\sigma t+R)\right]^{-1} F(t)^{2} 0, \quad t \geqq 0,
$$

since $J_{1}>0$. On the other hand, since $J_{2} \geqq 0,(3.5),(3.6)$ yield the auxiliary estimates

$$
\begin{gathered}
F^{\prime \prime}(t) \geqq B_{0}(\sigma t+R)^{-1}, \quad t>0, \quad F^{\prime}(t) \geqq \sigma^{-1} B_{0} \log \left(\frac{\sigma t+R}{R}\right), \quad t>0, \\
F(t) \geqq C \sigma^{-2} B_{0}(\sigma t+R) \log (\sigma t+R), \quad t>R_{1},
\end{gathered}
$$

since $F(0)=F^{\prime}(0)=0$. Equations $(3.9 \mathrm{a}, \mathrm{b}, \mathrm{c})$ are sufficient to prove blow-up. Indeed, if we let $s=\sigma t$ and $\widetilde{F}(s)=\sigma^{2} / \bar{\rho} F(t)$ we have

$$
\begin{aligned}
\widetilde{F}^{\prime \prime}(s) & \geqq C\left[(s+R)^{3} \log (s+R)\right]^{-1} \tilde{F}(s)^{2}, \\
\tilde{F}^{\prime}(s) & \geqq C \bar{\rho}^{-1} B_{0} \log (s+R), \\
\widetilde{F}(s) & \geqq C \bar{\rho}^{-1} B_{0}(s+R) \log (s+R),
\end{aligned}
$$


for $s>\left(R-R_{0}\right) / 2$. In [9], we showed that any $C^{2}$ function $\widetilde{F}(s)$ which satisfies the above inequalities has a finite life span bounded above by $C \exp \left[C \bar{\rho}^{2} / B_{0}^{2}\right]$ (cf. $42 \mathrm{a}-\mathrm{d}$ and the conclusion of the proof of Theorem 3 in [9]).

Hence, the life span of the $C^{1}$ solution is bounded above by

$$
\frac{C}{\sigma} \exp \left(C \bar{\sigma}^{2} / B_{0}^{2}\right)
$$

For the general case, $\gamma>1$, the adjustment occurs in (3.7) where now

$$
p-\bar{p}-\sigma^{2}(\rho-\bar{\rho}) \geqq A e^{\bar{S}}\left[\rho^{\gamma}-\bar{\rho}^{\gamma}-\gamma \bar{\rho}^{\gamma-1}(\rho-\bar{\rho})\right] \equiv A e^{\bar{S}} \Psi(\rho, \bar{\rho}) .
$$

By convexity of $\rho^{\gamma}, \Psi(\rho, \bar{\rho})$ is positive for $\rho \neq \bar{\rho}$. Using Taylor's theorem, we have the lower bound

$$
\psi(\rho, \bar{\rho}) \geqq C_{\gamma}(\bar{\rho}) \Phi_{\gamma}(\rho-\bar{\rho}),
$$

where $\Phi_{\gamma}(\cdot)$ is nonnegative, convex and

$$
\Phi_{\gamma}(a)= \begin{cases}a^{2}, & -\bar{\rho}<a<\bar{\rho} \\ a^{\gamma}, & 2 \bar{\rho}<a .\end{cases}
$$

Jensen's inequality is used in (3.8b). The resulting differential inequality still has a finite life span, although the upper bound will differ.

\section{Isentropic Flow and Weak Solutions}

The following corollary to Theorem 3 holds for weak solutions when the fluid is isentropic, that is, entropy is constant. Thus, the state equation becomes

$$
p=A e^{\bar{s}} \rho^{\gamma} \quad(A>0, \quad \gamma>1) \text {. }
$$

Corollary. A global weak solution $(\rho, u)$ of $\left(1.1 a, b, d^{\prime \prime}\right)$ (written in conservation form) with initial data $(1.2 a, b)$ which satisfies $(1.7 a, b)$ cannot be $C^{1}$ on the set

$$
D_{T}=\left\{(x, t): x \geqq R_{0}+\sigma t, 0 \leqq t \leqq T\right\},
$$

for all $T>0$. (Singularities come arbitrarily close to the wave front.)

Proof. Since $S \equiv \bar{S}$ is constant, inequality (3.10) holds without reference to the particle paths $x^{\prime}=u$. Therefore, the analysis of Theorem 3 is valid as long as the solution $(\rho, u)$ is $C^{1}$ in $D_{T}$, and we must conclude that $T$ is finite.

\section{References}

1. Courant, R., Friedrichs, K. O.: Supersonic flow and shock waves. New York: Interscience 1948

2. John, F.: Formation of singularities in one-dimensional nonlinear wave propagation. Commun. Pure Appl. Math. 27, 377-405 (1974)

3. John, F.: Blow-up of radial solutions of $u_{t t}=C^{2}\left(u_{t}\right) \Delta u$ in three space dimensions. To appear

4. Kato, T.: The Cauchy problem for quasilinear symmetric systems. Arch. Ration. Mech. Anal. 58, 181-205 (1975)

5. Klainerman, S. Majda, A.: Formation of singularities for wave equations including the nonlinear vibrating string. Commun. Pure Appl. Math. 33, 241-263 (1980) 
6. Klainerman, S. Majda, A.: Compressible and incompressible fluids. Commun. Pure Appl. Math. 35 , 629-651 (1982)

7. Lax, P.: Development of singularities of solutions of nonlinear hyperbolic partial differential equations. J. Math. Phys. 5, 611-613 (1964)

8. Liu, T.-P.: The development of singularities in the nonlinear waves for quasi-linear hyperbolic partial differential equations. J. Differ. Equations 33, 92-111 (1979)

9. Sideris, T.: Global behavior of solutions to nonlinear wave equations in three space dimensions. Commun. Partial Differ. Equations 8, 12, 1291-1323 (1983)

10. Sideris, T.: Formation of singularities of solutions to nonlinear hyperbolic equations. Arch. Ration. Mech. Anal. 86, 4, 369-381 (1984)

Communicated by L. Nirenberg

Received September 10, 1983; in revised form March 11, 1985 
